Las preguntas del docente en la interacción didáctica. PÁginas 15-28 en Revista de la Escuela de Ciencias de la EDUCACIÓN, AÑO12, NRO.11, VOL. 1, ENERO A JUNIO DE 2016. ISSN 1851-6297. ISSN EN LINEA 2362-3349.

\title{
LAS PREGUNTAS DEL DOCENTE EN LA INTERACCIÓN DIDÁCTICA
}

\author{
Rosana Alejandra Serafini ${ }^{1}$ \\ Universidad Argentina de la Empresa, Argentina. \\ rserafini@uade.edu.ar
}

Recibido: 11/02/2016 Aceptado: 23/05/2016

\section{Resumen}

El presente artículo está basado en una investigación de tipo cualitativa cuyo tema principal es "las interacciones discursivas en el aula universitaria".

En dicha investigación la pregunta central es por "el uso que hacen los docentes de la interrogación en la clase universitaria”. Caracterizar los tipos de interacción que se establecen entre docentes y estudiantes a través del uso que hacen los primeros de la interrogación y establecer relaciones ente los modos de preguntar del profesor y sus concepciones acerca del contenido y los procesos de enseñanza y aprendizaje, son los objetivos principales.

Se trata de un estudio descriptivo/interpretativo/explicativo, empleando una metodología cualitativa, un proceso inductivo, si bien aparece un marco teórico previo.

Sintéticamente, cuando los profesores dicen sentirse más seguros con los contenidos (docentes expertos), suelen formular preguntas con mayor facilidad con la intención de generar un dialogo genuino con los estudiantes en sus clases. Quiénes poseen menos experiencia, suelen trabajar con una modalidad más expositiva, casi sin formular preguntas ni abrir demasiado el espacio para las preguntas de sus estudiantes.

Pero no interesa que el docente "pregunte mucho" sino que pregunte de manera "provechosa" a los fines de la enseñanza y el aprendizaje.

\section{Palabras clave:}

Interacción - Didáctica - Discurso - Interrogación - Clase.

* Magister y Especialista en Didáctica. Facultad de Filosofía y Letras (UBA). Especialista en Psicopedagogía (GCBA - Secretaría de Salud) - Lic. en Cs. de la Educación - Facultad de Filosofía y Letras (UBA). Profesora Adjunta Ordinaria en el Depto. de Ciencias Sociales y Humanidades. Facultad de Ciencias Jurídicas y Sociales (UADE). Profesora en el Posgrado de Formación Docente de la Escuela de Educación (UADE) y Contenidista para la Escuela de Educación y UADE VIRT. 
Revista de la Escuela de Ciencias de la Educación, año 12, nRo.11, vol. 1, enero a junio de 2016. Páginas 15-28. ISSN 1851-6297. ISSN EN LÍNEA 2362-3349. LAS PREGUNTAS DEL DOCENTE EN LA INTERACCIÓN DIDÁCTICA. ROSANA AlejandRa SerafinI

\begin{abstract}
This article is based on a research of qualitative type whose main theme is "discourse interactions in the University classroom". In this research, the central question is "the use that make teachers in polling in the University class". It is: characterize the types of interaction established between teachers and students through the use that make the first polling and establish relations between modes of ask the Professor and his ideas about the content and processes of teaching and learning. It is a study descriptive/interpretative/explanatory, using a qualitative methodology, an inductive process, even though is a prior theoretical framework. In summary, in those classes where teachers say they feel safer with the contents (educational experts), tend to ask questions more easily with the aim of creating a genuine dialogue with the students. In those classes where teachers have less experience, they tend to work with a more expository manner, almost without questions or open space for students questions too.

But not interested in the teacher "ask much" that ask of "win-win" way for the purposes of teaching and learning.
\end{abstract}

Keywords:

Interaction - Didactic - Discourse - Polling - Class.

\title{
Introducción
}

El presente artículo intenta describir los resultados de un proceso de investigación realizado en el marco de mi tesis de Maestría en Didáctica de la Universidad de Buenos Aires, que se ha llevado a cabo durante los años 2011 y 2013 gracias a la colaboración de docentes universitarios, quiénes gentilmente abrieron las puertas de sus aulas permitiendo penetrar en el mundo de la interacción didáctica.

El trabajo de campo fue realizado en dos universidades: una pública y otra privada. Los profesores corresponden al área de las ciencias sociales (Sociología, Historia y Ciencia Política) a cargo de grupos de estudiantes de primero y segundo año de dichas carreras.

La pregunta por "el uso que hacen los docentes de la interrogación en la clase universitaria" constituye el interrogante principal de la tesis, con el afán de ahondar en el campo de la didáctica de nivel superior. El abordaje del tema intenta dar respuesta a la siguiente problemática: las concepciones que tienen los docentes acerca del uso de preguntas en clase y sus modos de preguntar tienen relación directa con sus concepciones acerca del contenido, del proceso de aprendizaje y de los propósitos de la enseñanza.

Dicho planteo se vio reflejado a través de las voces y la actuación de los docentes en clase y el análisis realizado en función de los datos obtenidos mediante el trabajo de campo y su relación con el marco teórico conceptual. 
Revista de la Escuela de Ciencias de la Educación, año 12, nRo. 11, vol. 1, enero a junio de 2016. Páginas 15-28. ISSN 1851-6297. ISSN EN LINEA 2362-3349. LAS PREGUNTAS DEL DOCENTE EN LA INTERACCIÓN DIDÁCTICA. ROSANA AleJANDRA SeRAFINI

De la problemática planteada inicialmente se desprendieron preguntas, que a su vez, son de dos tipos:

- Preguntas orientadas al análisis de la propia situación de interacción didáctica y el uso de la interrogación, que se analizaron mediante las observaciones de clase.

¿Cuál es el uso que hacen los profesores de las preguntas en clase? ¿Qué buscan con esas preguntas? ¿Cómo las formulan? ¿Cómo reciben las respuestas de los estudiantes? ¿Cuál es el modo con el que se dirigen a los mismos? ¿Qué modalidades de interacción establecen con sus estudiantes en clase?

- Preguntas vinculadas a las concepciones del docente con respecto al uso de la interrogación, que se analizaron mediante las entrevistas realizadas a los profesores, previa y post observación de clase.

¿En qué casos trabajan con preguntas y en qué casos no lo hacen? ¿Cuáles son los criterios seleccionados para trabajar con preguntas? ¿Cuáles son los propósitos que persiguen? ¿Las preguntas que formulan, son pensadas con anterioridad o improvisadas en el contexto de la clase?

\section{Interacciones discursivas en el aula universitaria}

\section{El uso que hacen los docentes de la interrogación}

La interacción en clase ha sido objeto de estudio para distintos autores. El entendimiento entre docentes y alumnos es importante para la mejora de la enseñanza y/o del aprendizaje, de aquí la importancia de observar la interacción didáctica, con el registro y análisis de los intercambios verbales o no verbales que se establecen entre unos y otros en el contexto de la clase. Comprender la interacción en el aula implica utilizar los hallazgos de investigaciones sociológicas y psico-sociológicas que permiten hacer comprensibles los procesos que se dan en la clase.

El tema fue abordado desde un enfoque interaccionista y didáctico, habida cuenta de la emergencia de la noción de "interacción" en numerosos campos de las ciencias humanas interesadas por este tema, representantes de una orientación epistemológica y metodológica que sigue los pasos de la etnografía.

Una "mirada interaccionista del aula universitaria" implica entonces examinar los recursos que profesores y alumnos pueden utilizar en cada interacción, así como sus actitudes, sus creencias, sus perspectivas, revisando cómo, entre ellos, "negocian un mundo compartido" (Delamont, 1984).

En el desarrollo conceptual de la tesis se hace referencia, en principio, a algunos supuestos acerca de la enseñanza en tanto acción e interacción social siguiendo la teoría de Max Weber, para vincularlo posteriormente con la teorización de Anthony Giddens acerca de las formas más estables de relación 
Revista de la Escuela de Ciencias de la Educación, año 12, nRo.11, vol. 1, enero a junio de 2016. Páginas 15-28. ISSN 1851-6297. ISSN EN LÍNEA 2362-3349. LAS PREGUNTAS DEL DOCENTE EN LA INTERACCIÓN DIDÁCTICA. ROSANA AlejandRa SerafinI

social. Luego, desde los trabajos de Jackson (1968), Delamont (1984) y Burbules (1999) se aborda específicamente la acción de enseñar con sus distintos momentos: preactivo, en tanto programación de la enseñanza; interactivo, en tanto interacción en clase; postactivo, qué sucede después de la enseñanza, la idea es adentrarse en el seno de la situación interactiva, su contexto, las disposiciones hacia la tarea tanto del docente como del alumno, la relación pedagógica. Finalmente se trabaja puntualmente el tema de las preguntas del docente en el aula: tipos de preguntas, modos de preguntar, propósitos que persigue el docente con el uso de la interrogación en clase.

En cuanto a lo metodológico, el trabajo se inscribe en una perspectiva cualitativa, tratándose de un proceso inductivo, que intenta basarse en los datos recogidos: emergencia de la teoría a partir del trabajo de campo, si bien aparece un marco teórico previo con el cuál ir a "mirar".

La metodología fue recoger datos a través del registro etnográfico/descripción densa, para luego analizarlos utilizando la hermenéutica como recurso al tratar de confrontar y poner en situación de análisis los datos que arrojaron las observaciones y las entrevistas con los docentes. Se estudiaron varias clases durante un cuatrimestre. El trabajo se realizó con ocho docentes.

Un estudio descriptivo/explicativo, según sus objetivos básicos. Descriptivo porque se propone describir las interacciones discursivas en el aula universitaria, la situación interactiva que se genera a través del uso de la interrogación por parte de los profesores. Explicativo porque buscar relacionar los fenómenos a estudiar: explicar las relaciones existentes entre el uso que hacen los docentes de la interrogación en clase y sus concepciones acerca del contenido a enseñar y de los procesos de enseñanza y aprendizaje.

En cuanto a las técnicas de recolección de datos el trabajo se organizó respetando la siguiente secuencia:

1) Fase exploratoria: entrevistas iniciales, en profundidad, previas a la observación de sus clases, para ver "qué piensan" los docentes con respecto al uso de la interrogación como herramienta didáctica.

2) Fase de profundización I: observaciones de clases, para ver "qué hacen" los docentes en la interacción.

3) Fase de profundización II: entrevistas a los mismos docentes, posteriores a la observación de sus clases, para recuperar aquellos elementos que pudieron no haberse indagado en la entrevista inicial o no haber resultado claros en las observaciones de clase.

En cuanto a la metodología de análisis de cada clase se estableció una fragmentación en cuatro momentos.

- Presentación, del docente y la observadora.

- Introducción del tema a tratar y de las estrategias de enseñanza.

- Desarrollo de la clase propiamente dicho. 
Revista de la Escuela de Ciencias de la Educación, año 12, nRo. 11, vol. 1, enero a junio de 2016. Páginas 15-28. ISSN 1851-6297. ISSN EN LINEA 2362-3349. LAS PREGUNTAS DEL DOCENTE EN LA INTERACCIÓN DIDÁCTICA. ROSANA AlejandRa SerafinI

- Cierre con despedida del docente y sugerencia de lecturas de material bibliográfico para la próxima clase, control de asistencia en algunas clases.

En relación al "clima de la clase", el análisis de la interacción se planteó en tres dimensiones.

- Participación de los estudiantes.

- Disposiciones del docente (gestos, tonos de voz del docente).

- Uso de “apoyos” para la enseñanza (recursos utilizados por el docente).

Con posterioridad a la recogida de información, se contabilizaron las preguntas que realizó cada docente en los distintos momentos de la clase, valiéndose del apoyo tecnológico que ofrece el programa ATLAS-TI. Luego se separaron y contabilizaron las preguntas abiertas y las preguntas cerradas, según la clasificación establecida por Anijovich y Mora (2009). Además se incluyeron preguntas que formularon los estudiantes. Finalmente se realizó el análisis y síntesis escrita de cada clase y de cada entrevista con los docentes.

Sin perder de vista el eje de la interacción en clase, se puso el foco en el diálogo docente - estudiantes y la participación de los estudiantes en clase. En esta dirección, se pudo ver que la mayoría de los profesores usa preguntas en clase, si bien lo hacen con diferentes intenciones: para generar un intercambio en el sentido de la interacción grupal, como herramienta de dialogo con sus estudiantes, para mantener el interés, para motivarlos, para involucrarlos en la clase, o con el propósito de "testear la información" en el sentido de la evaluación, o como herramienta para ejercer un "control de lectura".

De esta manera, cuando los profesores preguntan para fomentar el diálogo hay un modo de interacción que están proponiendo. Generar preguntas como modo interactivo, como herramienta para entablar un dialogo con los estudiantes nos conduce a pensar que el modelo de interacción clásico Interrogación - Respuesta - Evaluación (IRE), en algunos casos, va variando. Existen otras modalidades de la "interacción discursiva" que se van estableciendo a través del uso de la interrogación en clase y que se hallaron a través del trabajo de campo realizado: el docente pregunta para generar un debate (esquema radial), la retórica del docente como modo de interacción didáctica, el modo de interacción solemne y distante, el autocontrol del docente como modalidad de interacción y el "zigzagueo" de preguntas y respuestas en la interacción.

Sin embargo, cabe destacar que no todas las preguntas del docente en clase generan interacción. Algunos docentes usan la retórica para embellecer el discurso, como modo de auto-confirmación de sus ideas, con preguntas dirigidas a sí mismo, como estrategia didáctica planificada en algunos casos y en otros de manera espontánea, sin previa reflexión o programación. Además las "muletillas" del lenguaje, a veces enunciadas en forma de pregunta, tampoco generan interacción con los estudiantes; surge aquí una nueva categoría de 
Revista de la Escuela de Ciencias de la Educación, año12, nRo.11, vol. 1, enero a Junio de 2016. Páginas 15-28. ISSN 1851-6297. ISSN EN LINEA 2362-3349. LAS PREGUNTAS DEL DOCENTE EN LA INTERACCIÓN DIDÁCTICA. ROSANA AlejandRa SerafinI

preguntas, anclada en la detección de un tipo especial de interrogación que resulta un reaseguro para el docente. Podemos denominarlas "preguntas de auto-confirmación” (¿Si?, ¿Se entiende?, ¿Queda claro?). Son aquellas preguntas que tranquilizan al docente, que le proveen un reaseguro acerca de lo que está diciendo, no son preguntas de comprensión, se apoyan en las emociones del docente.

En dichas clases, surge también otra categoría de preguntas que llamaremos "sugeridas", porque proponen de antemano la respuesta que el docente espera que los estudiantes brinden, en muchos casos adelantando la respuesta u ofreciendo palabras, a modo de pistas, que sugieren abiertamente la respuesta. En la siguiente secuencia de una de las clases de Historia Argentina se puede notar tal afirmación.

Profesor: Unión Democrática. ¿Y cómo miraba Braden a Perón? ¿Cómo qué?

Estudiantes: (a coro) Como un fascista.

$P$ : Como un fascista. ¿Y después también como un...?

E: Nazi...

La pregunta siguiente es un ejemplo similar al anterior: ... ¿eso es lo que dice quién? Los estudiantes deben responder "quién" (alguien) dijo eso. Dichas preguntas suponen de antemano lo que deben responder los estudiantes, generando una respuesta breve y directa.

Otro tipo de preguntas que se desprende del análisis de las clases y crea una nueva categoría, son las que podemos denominar preguntas incompletas, porque sus enunciados quedan inconclusos en la formulación y se asemejan a adivinanzas, por ejemplo: ¿Y después también como un...? Son preguntas sugeridas, del subtipo de preguntas incompletas.

En una de las entrevistas posteriores a las observaciones de clase, una profesora propone una nueva denominación para cierto tipo de preguntas: las preguntas-problema. Su formulación puede tener distintas intenciones: generar el análisis o el debate sobre algún tema, en este caso las preguntas de la profesora sirven como "disparadores" para generar un intercambio con los estudiantes en el sentido de la interacción grupal; "despabilar", cuando las clases comienzan por la mañana muy temprano y se hace necesario motivar a los estudiantes utilizando herramientas que los conecten con el contenido de la materia; comprometerlos con la materia y con el tema de la clase del día. El propósito que la docente persigue es usar preguntas como una herramienta de diálogo, preguntas que van más allá de la veracidad de las respuestas de los estudiantes. El objetivo es dialogar, conocerlos, tener una imagen del grupo, medir el grado de interés por la materia o el tema, construir los contenidos con los estudiantes más que corroborar si han leído los textos para la clase del día. Tiene privilegio este tipo de preguntas en el desarrollo de la clase porque 
Revista de la Escuela de Ciencias de la Educación, año12, nRo.11, vol. 1, enero a Junio de 2016. Páginas 15-28. ISSN 1851-6297. ISSN EN LINEA 2362-3349. LAS PREGUNTAS DEL DOCENTE EN LA INTERACCIÓN DIDÁCTICA. ROSANA Alejandra SerafinI

son las que intentan generar un debate sobre el tema tratado o un análisis del mismo y para ello es necesario que la clase se encuentre plenamente en tarea. Implica concepciones amplias, flexibles, críticas y cuestionadoras por parte del docente acerca de la enseñanza y el aprendizaje; son preguntas que buscan problematizar el objeto de estudio confiando en la capacidad de los estudiantes para lograrlo. Son preguntas que intentan que los estudiantes pregunten y cuestionen, en contra de la aceptación pasiva del contenido.

En todas las clases observadas se logró establecer una relación directa entre el clima de la clase y el favorecimiento de determinado tipo de preguntas por parte del docente: las preguntas sencillas de organización de la clase y las preguntas sugeridas. Las primeras parecieran "abrir el dialogo" y se vinculan al concepto de tarea académica (1), especialmente a un tipo de tarea académica que se conoce como "procedimiento de rutina" y se refiere a seguir pasos o aplicar reglas para resolver un problema (Doyle, 1983). El modo de intervención del docente en cada caso y el tipo de tareas que propone a los estudiantes responden a este concepto. El segundo tipo de preguntas, que hemos llamado sugeridas, parecen "mantener el dialogo" entre docentes y estudiantes durante el devenir de la clase.

Por otro lado, los profesores que más preguntan no generan necesariamente mejores interacciones con sus estudiantes. Se observó que en algunas clases los mismos formulan pocas preguntas pero de muy buena calidad, con ello generan un intercambio fluido y dinámico con los estudiantes. Otras clases están "llenas" de preguntas por parte del docente pero éstas no colaboran en el establecimiento de un dialogo con los estudiantes. El interrogante en este caso sería: ¿el uso de preguntas en clase por parte del docente garantiza mejores procesos de enseñanza? La respuesta es NO. Para que así sea, las preguntas tienen que tener cierto tipo de formulación.

En tal sentido, los dos tipos de pregunta que generan mejores procesos interactivos entre docentes y estudiantes son: las preguntas sencillas orientadas al contenido disciplinar y las preguntas de comprensión. Las mismas generan un intercambio fluido. De tal manera, podemos pensar que al fin y al cabo no interesa que el docente "pregunte mucho" sino que pregunte de manera "provechosa" a los fines de la enseñanza y el aprendizaje.

Se nota además, en varios docentes, cierta dificultad para esperar la respuesta de los alumnos frente a la enunciación de sus preguntas. Dificultad para tolerar el "silencio" que puede devenir luego de una pregunta, mientras los estudiantes piensan y elaboran una respuesta. En general los profesores pretenden una respuesta rápida y si es posible, acertada, para continuar con la dinámica de la clase. Dicha situación puede jugar en contra de una buena interacción entre docentes y estudiantes.

El cuadro que se presenta a continuación muestra una tipología "construida" de preguntas analizadas: 
Revista de la Escuela de Ciencias de la Educación, año 12, nRo.11, vol. 1, enero a Junio de 2016. Páginas 15-28. ISSN 1851-6297. ISSN EN LINEA 2362-3349. LAS PREGUNTAS DEL DOCENTE EN LA INTERACCIÓN DIDÁCTICA. ROSANA AleJANDRA SerafinI

\begin{tabular}{|c|c|c|}
\hline $\begin{array}{l}\text { Tipos de } \\
\text { pregunta }\end{array}$ & Características & $\begin{array}{l}\text { Momento de la } \\
\text { clase en que se } \\
\text { utilizan }\end{array}$ \\
\hline Retóricas & $\begin{array}{l}\text { Son preguntas cerradas, útiles para } \\
\text { verificar un acuerdo, o bien, son } \\
\text { empleadas como recurso retórico para } \\
\text { embellecer la expresión en el devenir de } \\
\text { una conversación. }\end{array}$ & $\begin{array}{l}\text { Presentación } \\
\text { Introducción } \\
\text { Desarrollo }\end{array}$ \\
\hline $\begin{array}{l}\text { Sencillas, de } \\
\text { organización } \\
\text { de la clase }\end{array}$ & $\begin{array}{l}\text { Son preguntas que requieren respuestas } \\
\text { breves, únicas, información precisa. } \\
\text { Promueven respuestas simples. }\end{array}$ & $\begin{array}{l}\text { Presentación } \\
\text { Introducción } \\
\text { Cierre }\end{array}$ \\
\hline $\begin{array}{l}\text { Sencillas, } \\
\text { orientadas } \\
\text { al contenido } \\
\text { disciplinar }\end{array}$ & $\begin{array}{l}\text { Son preguntas que requieren respuestas } \\
\text { breves, únicas, información precisa pero } \\
\text { con respecto al contenido de la disciplina } \\
\text { en cuestión. También promueven } \\
\text { respuestas simples. }\end{array}$ & $\begin{array}{l}\text { Desarrollo } \\
\text { Cierre }\end{array}$ \\
\hline $\begin{array}{l}\text { De mediana } \\
\text { complejidad } \\
\text { o de } \\
\text { comprensión }\end{array}$ & $\begin{array}{l}\text { Son preguntas que se proponen } \\
\text { estimular el procesamiento de la } \\
\text { información. Se usan para indagar con } \\
\text { cierto grado de profundidad. El alumno } \\
\text { necesita pensar, relacionar datos, } \\
\text { clasificar, comparar, para elaborar su } \\
\text { respuesta. }\end{array}$ & Desarrollo \\
\hline $\begin{array}{l}\text { Complejas } \\
\text { o de orden } \\
\text { cognitivo } \\
\text { superior }\end{array}$ & $\begin{array}{l}\text { Son preguntas que demandan } \\
\text { respuestas que exigen interpretar, } \\
\text { predecir, evaluar críticamente. }\end{array}$ & Desarrollo \\
\hline $\begin{array}{l}\text { Metacog- } \\
\text { nitivas }\end{array}$ & $\begin{array}{l}\text { Son preguntas que se proponen ayudar } \\
\text { a los estudiantes a reflexionar sobre } \\
\text { su modo de aprender y de pensar. } \\
\text { Demandan que los estudiantes analicen y } \\
\text { revisen cómo estudian, cómo interpretan } \\
\text { y resuelven sus tareas, qué dificultades } \\
\text { encuentran, cuáles son sus fortalezas, } \\
\text { qué ayuda necesitan. }\end{array}$ & $\begin{array}{l}\text { En ningún } \\
\text { momento }\end{array}$ \\
\hline
\end{tabular}

[Continúa en pág.23] 
Revista de la Escuela de Ciencias de la Educación, año 12, nRo. 11, vol. 1, enero a Junio de 2016. Páginas 15-28. ISSN 1851-6297. ISSN EN LINEA 2362-3349. LAS PREGUNTAS DEL DOCENTE EN LA INTERACCIÓN DIDÁCTICA. ROSANA Alejandra SerafinI

[Viene de pág.22]

\begin{tabular}{|l|l|l|}
\hline $\begin{array}{l}\text { Tipos de } \\
\text { pregunta }\end{array}$ & Características & $\begin{array}{l}\text { Momento de la } \\
\text { clase en que se } \\
\text { utilizan }\end{array}$ \\
\hline $\begin{array}{l}\text { De autocon- } \\
\text { firmación }\end{array}$ & $\begin{array}{l}\text { Son preguntas que actúan muchas } \\
\text { veces como "muletillas" del lenguaje. } \\
\text { Son aquellas que tranquilizan al docente, } \\
\text { que le proveen un reaseguro acerca de } \\
\text { lo que está diciendo, no son preguntas } \\
\text { de comprensión, sino preguntas de } \\
\text { "emocionalidad". }\end{array}$ & $\begin{array}{l}\text { Introducción } \\
\text { Presentación } \\
\text { Desarrollo } \\
\text { Cierre }\end{array}$ \\
\hline Sugeridas & $\begin{array}{l}\text { Son preguntas que sugieren de } \\
\text { antemano la respuesta que el docente } \\
\text { espera que los estudiantes brinden, en } \\
\text { muchos casos adelantando la respuesta } \\
\text { u ofreciendo palabras que sugieren } \\
\text { abiertamente la respuesta. }\end{array}$ & Desarrollo \\
\hline Incompletas & $\begin{array}{l}\text { Son preguntas que se asemejan a } \\
\text { adivinanzas porque sus enunciados son } \\
\text { incompletos en su formulación. }\end{array}$ & Desarrollo \\
\hline
\end{tabular}

\section{Reflexiones finales}

En la tipología presentada hay preguntas cuyo uso se omite: son las preguntas metacognitivas. Por tratarse de preguntas que pretenden que los estudiantes analicen cómo interpretan y resuelven sus tareas, qué dificultades se les presentan en el proceso de resolución de problemas, qué fortalezas o debilidades encuentran en sus propios procesos de aprendizaje y qué tipo de ayuda necesitan, demandan al docente una actitud distinta a lo esperado convencionalmente. El uso de tales preguntas en la interacción didáctica exige una predisposición distinta por parte del profesor, se requiere un esfuerzo aún mayor en el transcurso de la clase, atendiendo no solamente al nivel de comprensión de los estudiantes en el momento de la interacción en clase sino también sus modos de aprender y de pensar previos a la interacción.

Las concepciones de los docentes sobre el uso de preguntas en la enseñanza se vinculan con sus propias concepciones acerca de los procesos de enseñanza y aprendizaje y sus concepciones sobre los contenidos a enseñar. La tipología de preguntas construida sobre las evidencias obtenidas en las entrevistas, previas y posteriores a la observación de sus clases, da cuenta de ello. 
Revista de la Escuela de Ciencias de la Educación, año12, nRo.11, vol. 1, enero a Junio de 2016. Páginas 15-28. ISSN 1851-6297. ISSN EN LINEA 2362-3349. LAS PREGUNTAS DEL DOCENTE EN LA INTERACCIÓN DIDÁCTICA. ROSANA AlejandRa SerafinI

Hay coincidencia en la mayoría de los docentes en cuanto a "lo que dicen que hacen" en sus clases y "lo que realmente hacen" en la interacción a través del uso de la interrogación. Aquellas concepciones que aparecen de manera implícita en el discurso del profesor en las entrevistas, luego se hace explícito en la clase. En un solo caso se encontró diferencia entre "lo dicho" en las entrevistas y "lo hecho" en clase: la intención explícita de la profesora en las entrevistas es que la clase no sea estrictamente expositiva pero en la interacción con los estudiantes este planteo entra en contradicción.

Se observa una clase donde priman las preguntas retóricas y la modalidad de interacción resulta expositiva, magistral. Dichas preguntas surgen cuando se nota cierta dificultad por parte de la docente para esperar la respuesta de los estudiantes, para tolerar el silencio que deviene luego de una pregunta, mientras los estudiantes piensan las posibles respuestas. Igual situación se observa en otros profesores, pero ello queda explícito en las entrevistas previas a la observación.

Desde una perspectiva psico-sociológica, se observa que en el aula de estos docentes los roles del profesor y del estudiante en la clase están bien definidos anticipadamente. Los docentes inician su clase "solicitando" y los estudiantes "responden" en consecuencia; luego los docentes aprueban o no, siendo ésta la modalidad de interacción que más se observa (Modelo IRE). La mayor parte de dichas "solicitudes" se dan en forma de "preguntas".

La posición de los estudiantes frente a los distintos tipos de preguntas surge de las observaciones de clase. El foco está puesto en las interacciones que se promueven en clase, dando respuesta al objetivo de comprender los intercambios verbales entre docentes y estudiantes y conocer cuáles son los propósitos que persiguen los docentes con las preguntas.

El siguiente cuadro sintetiza las respuestas de los estudiantes, frente a los diferentes tipos de preguntas del profesor:

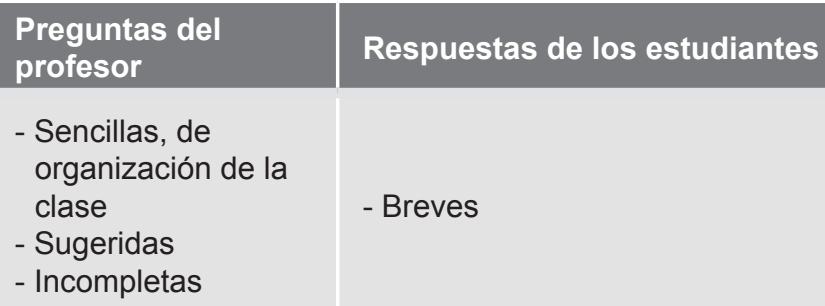

- Sencillas, de organización de la clase - Breves

- Sugeridas

- Incompletas

- Directas 
Revista de la Escuela de Ciencias de la Educación, año12, nRo.11, vol. 1, enero a Junio de 2016. Páginas 15-28. ISSN 1851-6297. ISSN EN LINEA 2362-3349. LAS PREGUNTAS DEL DOCENTE EN LA INTERACCIÓN DIDÁCTICA. ROSANA ALEJANDRA SERAFINI

[Viene de pág. 24]
Preguntas del
profesor
Respuestas de los estudiantes
- De comprensión, de mediana
- Informativas
complejidad

- Exposiciones de una manera de ver las cosas

- Relatos de experiencias propias

- De comprensión, de mediana complejidad

- Otra Pregunta como verificación de lo aprendido o

- Sencillas orientadas bien para aclarar dudas al contenido disciplinar

- Complejas, de orden cognitivo superior

- Intentos de satisfacer una indagación

- Fundamentación de sus propias ideas

- Exposiciones de una manera de ver las cosas

- Interpretación de las ideas del autor que están estudiando

Se ha visto que a los estudiantes les cuesta preguntar, salvo en alguna de las clases observadas donde el tipo de información que traen los mismos a clase y nivel de lectura de textos parece bueno, entonces se atreven a preguntar; pero la tendencia es más bien ejercer un rol pasivo y receptivo, esperar que el docente proponga, presente, enuncie, muestre, pregunte.

Sólo en una de las clases observadas los estudiantes formulan muchas preguntas. A veces como respuesta, otras veces preguntan para aclarar dudas o recabar información más precisa. También surgen preguntas de autoconfirmación por parte de los estudiantes, de reaseguro, que promueven en los profesores respuestas precisas y breves. En este caso, las preguntas de 
Revista de la Escuela de Ciencias de la Educación, año12, nRo.11, vol. 1, enero a Junio de 2016. Páginas 15-28. ISSN 1851-6297. ISSN EN LINEA 2362-3349. LAS PREGUNTAS DEL DOCENTE EN LA INTERACCIÓN DIDÁCTICA. ROSANA AlejandRa SerafinI

los estudiantes no hacen más que reflejar la necesidad de éstos de buscar en la palabra del docente "la palabra sabia".

En los procesos de interacción en clase se ponen de manifiesto actitudes de los jóvenes, las que sintetizan en los sujetos individuales el proceso de influencia social. Se ha observado así que la modalidad de interacción que se establece en cada clase, centrada en los distintos aspectos que se fueron trabajando en esta tesis, son un indicador de quién es el que posee el poder en la clase y cómo los estudiantes responden en consecuencia.

Según los datos relevados a través de las entrevistas, en aquellas clases donde los profesores dicen sentirse más seguros con los contenidos, por su experiencia o su estilo personal, la posibilidad de formular preguntas aumenta con la intención de generar un dialogo genuino con los estudiantes. A veces, dicha situación se presenta sin necesidad de planificación previa, dando paso a una verdadera construcción del conocimiento junto a sus alumnos. Quiénes poseen menos experiencia, en cambio, suelen trabajar con una modalidad más expositiva, que muchos aún llaman "magistral", sin abrir demasiado el espacio para las preguntas, como una manera de tener más controlado el auditorio.

Vale la pena detenerse en la atención que prestan a las preguntas autores como Anijovich, Mora, Cols, Burbules, Delamont, por los beneficios que las mismas podrían otorgar a los estudiantes. En tal caso, si el hecho de trabajar con preguntas persigue propósitos tales como "promover la capacidad y disposición de los estudiantes para ser ellos mismos interrogadores cada vez más eficaces", ¿podríamos hablar de resultados pedagógicos valiosos?

\section{Notas Bibliográficas}

(1) Doyle (1983) dice que las tareas académicas deben clasificarse en: memoria, procedimientos de rutina, comprensión y opinión.

\section{Referencias Bibliográficas}

- Amidon, E. J. y Hunter, E. (1966). Improving teaching. Analysing verbal interaction in the classroom. New York: Rinehart and Winston

- Anguerra, M. T. (1978). Metodología de la observación en las Ciencias Humanas. Madrid, Cátedra, pp. $118-119$.

- $\quad$ Anijovich, R. y Mora, S., (2009). Estrategias de enseñanza. Buenos Aires: Aique.

- Ardoino, J. (1974). "Prefacio" a la obra de Lobrot, Pedagogía Institucional. La escuela hacia la autogestión. Buenos Aires: Humanitas.

- $\quad$ Astolfi, J.P. (1993). Tres paradigmas para la investigación en Didáctica en Revue Francaise de Pedagogie, № 103 (Traducción a cago de Residencia de Traducción I.E.S. en Lenguas Vivas - UBA).

- Batallán, G. (1982). Talleres de educadores como modalidad de perfeccionamiento operativo. Informe de investigación, CIE, tomos I y II. 
Revista de la Escuela de Ciencias de la Educación, año12, nRo.11, vol. 1, enero a Junio de 2016. Páginas 15-28. ISSN 1851-6297. ISSN EN LINEA 2362-3349. LAS PREGUNTAS DEL DOCENTE EN LA INTERACCIÓN DIDÁCTICA. ROSANA AlejandRa SerafinI

- Batallán, G. (1999). La aproximación de la etnografía para la investigación educacional. Reflexiones sobre su uso reciente en Argentina y Chile. En Revista del IICE, Año VIII, № 14, pp. 3-11.

- Burbules, N. (1999). El diálogo en la enseñanza. Teoría y práctica. Buenos Aires: Amorrortu.

- $\quad$ Candela, A. (1991). Transformaciones del conocimiento científico en el aula. En Rockwell E., La escuela cotidiana, (cap. 5, pp. 173-197). México: Centro de Investigación y de Estudios Avanzados del Instituto Politécnico Nacional.

- Candela, A. (2001). Corrientes teóricas sobre discurso en el aula. Revista Mexicana de Investigación Educativa, vol. 6, № 12, pp. 317-333.

- Candela, A., Rockwell, E., Coll, C. (2009). ¿Qué demonios pasa en las aulas? La investigación cualitativa del aula. CPU-e, Revista de Investigación Educativa, 8. Recuperado el 15 de enero de 2010, de http://www.uv.mx/cpue/num8/inves/candela_rockwell_coll_aulas.html

- Cazden, C. B. (1991). El discurso en el aula. El lenguaje de la enseñanza y del aprendizaje. Madrid: Paidós

- Cols, E. y Basabe, L. (2007). La enseñanza. En Camilloni, A. et al, El saber didáctico. Buenos Aires: Paidós.

- $\quad$ Delamont, S. (1984). La interacción didáctica. Madrid: Cincel-Kapelusz.

- Doyle, W. (1983). El trabajo académico. Revisión de la Investigación Educativa. 53, 159-199.

- Edelstein G., Coria A. (1995). Imágenes e imaginación. Iniciación a la docencia. Buenos Aires: Kapelusz.

- Edwards, V. et al (1993). Prácticas de trabajo y socialización en establecimientos de educación media, Informe final, PIIE, Universidad Católica de Temuco y Universidad de La Serena, Santiago, Chile.

- Ellsworth, E. (1977). Posiciones en la enseñanza. Madrid: Akal.

- Fenstermacher, G. (1989). Tres aspectos de la filosofía de la investigación sobre la enseñanza. Barcelona: Editorial Paidós.

- Freire, P. y Faundez, A. (1985). Por una pedagogía de la pregunta. Crítica a una educación basada en respuestas a preguntas inexistentes. Bs. As.: Siglo Veintiuno Editores (2013).

- Gibaja, R. (1994). El diseño y las etapas de investigación, Documento de Cátedra, Investigación y Estadística Educacional I, FFyL, UBA, Gibaja, R, (1987) La investigación en Educación. Discusiones y Alternativas, Cuaderno № 3, Centro de Investigaciones en Ciencias de la Educación, Bs. As.

- $\quad$ Giddens, A. (1971). El capitalismo y la moderna teoría sociológica. 2a edición: 1985. Barcelona: Ed. Labor.

- $\quad$ Giddens, A. (2004). Sociología. Madrid: Alianza.

- Gimeno Sacristán, J., y Pérez Gómez, A. I. (1995). Comprender y transformar la enseñanza. Madrid: Morata.

- Gimeno Sacristán, J. y Pérez Gómez, A. I. (1983). La enseñanza: su teoría y su práctica. Barcelona: Akal.

- Goetz, J.P. y Le Compte, M.D. (1984). Etnografía y diseño cualitativo en investigación educativa. Madrid: Morata.

- Gowin D. B. (1981). Hacia una teoría de la educación. Bs. As.: Ed. Aragón. (1985)

- Gouldner, A. (1979). La crisis de la sociología occidental. Bs. As.: Amorrortu.

- Jackson P. (1991). La vida en las aulas. Madrid: Morata. 
Revista de la Escuela de Ciencias de la Educación, año12, nRo.11, vol. 1, enero a Junio de 2016. Páginas 15-28. ISSN 1851-6297. ISSN EN LÍNEA 2362-3349. LAS PREGUNTAS DEL DOCENTE EN LA INTERACCIÓN DIDÁCTICA. ROSANA ALEJANDRA SERAFINI

- Litwin, E. (1997). Las configuraciones didácticas. Una nueva agenda para la enseñanza superior. Bs. As.: Paidós (2008)

- Mercer, N. (1999). Reflexiones sobre la investigación educativa y la educación a distancia (entrevista). En Revista del IICE, Año VIII, № 14, pp. 87-89.

- Pérez Gómez, A. I. (1983). Paradigmas contemporáneos de investigación didáctica. En Gimeno Sacristán, J. y Pérez Gómez, A. I. (Comp.) La enseñanza, su teoría y su práctica. Madrid: Akal.

- Rockwell, E. y Ezpeleta, J. (1983a). Escuela y clases subalternas, en Cuadernos Políticos, $\mathrm{N}^{\circ} 37$, ERA, México.

- Sarason, S. (2002). La enseñanza como arte de representación, Buenos Aires: Amorrortu.

- Simmel, G. (1986) Sociología. Estudios sobre las formas de asociación. Editorial Alianza.

- Simon, A. y Boyer, E. G. (1974). Mirrors for behaviour: an anthology of classroom observation instruments. Wyncote, P.A.: Communication Material Center.

- Souto, M. (1993). Hacia una didáctica de lo grupal. Buenos Aires: Miño y Dávila.

- Sverdlick, I. comp. (2007). La investigación educativa. Una herramienta de conocimiento y de acción. Buenos Aires: Noveduc.

- Tardif, M. (2004). Los saberes del docente y su desarrollo profesional. Madrid: Narcea.

- Taylor, S.J. y Bogdan, R. (1984). Introducción a los métodos cualitativos de investigación. Primera parte. España: Paidos.

- $\quad$ Touraine, A. (1984). El regreso del actor. Bs. As.: Eudeba. 1987.

- Weber, M. (1922). Economía y Sociedad. Esbozo de una Sociología Comprensiva, 2 tomos, $2^{\mathrm{a}}$ reimpresión, 1974. México: FCE.

- Weber, M. (1973) Ensayos sobre metodología sociológica. Buenos Aires: Amorrortu. 